\title{
Digging for historical data on the occurrence of benthic macrofaunal species in the southeastern Mediterranean
}

\author{
Irini Tsikopoulou $\ddagger$, Aglaia Legaki§, Panagiotis D. Dimitriou ${ }^{\ddagger}$ Evangelia Avramidoul, Nicolas Bailly", \\ Stamatina Nikolopoulouा \\ ‡ University of Crete, Heraklion, Greece \\ § University of Ahtens, Athens, Greece \\ | Aristotle University of Thessaloniki, Thessaloniki, Greece \\ I Institute of Marine Biology, Biotechnology and Aquaculture, Hellenic Centre for Marine Research (HCMR), Heraklion, \\ Greece
}

Corresponding author: Irini Tsikopoulou (fellow_irene@yahoo.gr), Stamatina Nikolopoulou (snikolo@hcmr.gr) Academic editor: Christos Arvanitidis

Received: 02 Aug 2016 | Accepted: 25 Aug 2016 | Published: 01 Nov 2016

Citation: Tsikopoulou I, Legaki A, Dimitriou P, Avramidou E, Bailly N, Nikolopoulou S (2016) Digging for historical data on the occurrence of benthic macrofaunal species in the southeastern Mediterranean. Biodiversity Data Journal 4: e10071. https://doi.org/10.3897/BDJ.4.e10071

\begin{abstract}
\section{Background}

The benthic macrofaunal biodiversity of the southeastern Mediterranean is considerably understudied compared to other Mediterranean regions. Monitoring biodiversity in this area is crucial as this region is particularly susceptible to biological invasions and temperature alteration. Historical biodiversity data could provide a useful baseline for monitoring potential changes and provide informarion to support a better understanding of the possible effects of anthropogenic activities on marine benthic communities.
\end{abstract}




\section{New information}

In this study, performed under the LifeWatchGreece Research Infrastructure, we present historical benthic occurrence data obtained from the sampling expedition carried out in 1933 by Adolf Steuer in the coastal area around Alexandria, Egypt, eastern Mediterranean. The occurrences were geo-referenced to more than 170 stations, mostly located in the area of Alexandria, and the nearby coasts and lakes. All records were digitized and species names were cross-checked and taxonomically updated using the World Register of Marine Species. The outcome clearly shows that such initiatives can reveal an unexpected amount of highly valuable biodiversity information for "data-poor" regions.

\section{Keywords}

marine biodiversity, macrofauna, Egypt expedition, eastern Mediterranean, historical dataset, data digitization, data rescue

\section{Introduction}

At the beginning of the 20th century, the importance of recording marine biodiversity was already recognized. Numerous expeditions had been organized with the aim of investigating "local fauna and flora" in various areas of the world. In 1924, Cambridge Expedition at the Suez Canal recorded the fauna of the Red Sea (Fox 1926), while Danish Oceanographical Expedition in 1908-1910 provided biological and hydrographical information for the Mediterranean and Adjacent Seas (Schmidt 1912). During these scientific expeditions, local biodiversity of various taxonomic groups was collected, recorded and the outcome was published in many scientific volumes. These historical occurrence data could provide a useful baseline for monitoring potential alterations, although they are often fragmented and found only in hard copy and grey literature. Such information is invaluable and needs to be digitized as it can provide the historical context for present observations and facilitate the process of setting correct reference conditions (Borja et al. 2012); it can also support predictive modeling of the consequences of human activities for the environment and biodiversity (Costello et al. 2013a). Additionally, historical datasets often contain descriptions of new species that are important for taxonomy as the first description of a species has legal priority for the name of this species (Costello et al. 2013b).

In this study, we present occurrence data which were digitized from 14 publications on the Egypt Expedition under the general report "The fishery grounds near Alexandria" made by Adolf Steuer and his colleagues and published between 1935-1940. Twelve of these publications included occurrence data on twelve macrofaunal groups and two of them were preliminary reports which described the sampling protocols that were followed during the expedition (Table 1). The digitization of "The fishery grounds near Alexandria" - Egypt 
Expedition - is a part of a broader strategy for the LifeWatchGreece Research Infrastructure, which aims at the digitization of historical datasets that contain biodiversity information from the Mediterranean region.

Table 1.

Taxa and related published volumes and information included in "The fishery grounds near Alexandria" dataset

\begin{tabular}{|c|c|c|c|c|c|c|}
\hline $\begin{array}{l}\text { Taxon name in } \\
\text { original } \\
\text { dataset }\end{array}$ & $\begin{array}{l}\text { Accepted } \\
\text { Taxon name }\end{array}$ & Volume & Author & Date & Title & Download URL \\
\hline- & - & 8 & A.Steuer & 1935 & $\begin{array}{l}\text { I. - Preliminary } \\
\text { report }\end{array}$ & $\begin{array}{l}\text { http://ipt.medobis.eu/ } \\
\text { resource? } \\
\underline{\text { r=egyptexpeditionprelim }} \\
\text { inaryrepor }\end{array}$ \\
\hline- & - & 9 & A. Vatova & 1935 & $\begin{array}{l}\text { II. A bottom } \\
\text { sample taken at } \\
\text { Alexandria }\end{array}$ & $\begin{array}{l}\text { http://ipt.medobis.eu/ } \\
\text { resource? } \\
\underline{\text { r=egyptexpeditionbotto }} \\
\underline{\text { msampler }}\end{array}$ \\
\hline Cirripeds & Cirripedia & 10 & H. Broch & 1935 & III. - Cirrepeds & $\begin{array}{l}\text { http://ipt.medobis.eu/ } \\
\text { resource? } \\
\underline{\text { r=egyptexpeditioncirripe }} \\
\underline{\text { ds }}\end{array}$ \\
\hline Hydroidea & Hydrozoa & 13 & A. Billard & 1936 & VI. - Hydroidea & $\begin{array}{l}\text { http://ipt.medobis.eu/ } \\
\text { resource? } \\
\text { r=egyptexpeditionhydroi } \\
\underline{\text { dea }}\end{array}$ \\
\hline Decapoda & Decapoda & 15 & H. Balss & 1936 & VII. Decapoda & $\begin{array}{l}\underline{\text { http://ipt.medobis.eu/ }} \\
\text { resource? } \\
\underline{\text { r=egyptexpeditiondecap }} \\
\underline{\text { oda }}\end{array}$ \\
\hline Pantopoda & Pantopoda & 16 & H. Helfer & 1936 & VIII. - Pantopoda & $\begin{array}{l}\text { http://ipt.medobis.eu/ } \\
\text { resource? } \\
\text { r=egyptexpeditionpanto } \\
\text { poda }\end{array}$ \\
\hline Amphipoda & Amphipoda & 18 & $\begin{array}{l}\text { A. } \\
\text { Schellenberg }\end{array}$ & 1936 & $\begin{array}{l}\text { X. - Amphipoda } \\
\text { Benthonica }\end{array}$ & $\begin{array}{l}\text { http://ipt.medobis.eu/ } \\
\text { resource? } \\
\text { r=egyptexpeditionamphi } \\
\text { poda }\end{array}$ \\
\hline
\end{tabular}




\begin{tabular}{|c|c|c|c|c|c|c|}
\hline $\begin{array}{l}\text { Annelides } \\
\text { Polychetes }\end{array}$ & Polychaeta & 19 & P. Fauvel & 1937 & $\begin{array}{l}\text { XI. - Annelides } \\
\text { Polychetes }\end{array}$ & $\begin{array}{l}\underline{\text { http://ipt.medobis.eu/ }} \\
\text { resource? } \\
\underline{\text { r=egyptexpeditionpolyc }} \\
\underline{\text { haeta }}\end{array}$ \\
\hline Echinoderma & Echinodermata & 21 & $\begin{array}{l}\text { Th. Mortensen } \\
\text { \& Ad. Steuer }\end{array}$ & 1937 & $\begin{array}{l}\text { XIII. - } \\
\text { Echinoderma }\end{array}$ & $\begin{array}{l}\underline{\text { http://ipt.medobis.eu/ }} \\
\text { resource? } \\
\underline{\text { r=egyptexpeditionechin }} \\
\underline{\text { oderma }}\end{array}$ \\
\hline $\begin{array}{l}\text { Cumacea, } \\
\text { Stomatopoda, } \\
\text { Leptostraca }\end{array}$ & $\begin{array}{l}\text { Cumacea, } \\
\text { Stomatopoda, } \\
\text { Leptostraca }\end{array}$ & 26 & A. Steuer & 1938 & $\begin{array}{l}\text { XVI. Cumacea, } \\
\text { stomatopoda, } \\
\text { leptostraca }\end{array}$ & $\begin{array}{l}\underline{\text { http://ipt.medobis.eu/ }} \\
\text { resource? } \\
\text { r=egyptexpeditioncuma } \\
\underline{\text { ceastomatopodaleptost }} \\
\underline{\text { acaea }}\end{array}$ \\
\hline Ascidiacea & Ascidiacea & 28 & H. Harant & 1939 & XVii. Ascidiacea & $\begin{array}{l}\underline{\text { http://ipt.medobis.eu/ }} \\
\underline{\text { resource? }} \\
\text { r=egyptexpeditionascidi } \\
\underline{\text { acea }}\end{array}$ \\
\hline $\begin{array}{l}\text { Sipunculoidea, } \\
\text { Phoronidea, } \\
\text { Brachiopoda, } \\
\text { Enteropneusta, } \\
\text { Arcania }\end{array}$ & $\begin{array}{l}\text { Sipuncula, } \\
\text { Phoronida, } \\
\text { Brachiopoda, } \\
\text { Enteropneusta, } \\
\text { Arcania }\end{array}$ & 30 & A. Steuer & 1939 & $\begin{array}{l}\text { XVIII. - } \\
\text { Sipunculoidea, } \\
\text { Phoronidea, } \\
\text { Brachiopoda, } \\
\text { Enteropneusta } \\
\text { and Arcania }\end{array}$ & $\begin{array}{l}\underline{\text { http://ipt.medobis.eu/ }} \\
\underline{\text { resource? }} \\
\underline{\text { r=egyptexpeditionsipun }} \\
\text { culoideaphoronidaebrac } \\
\underline{\text { hiopodaenteropneustaa }} \\
\text { crania }\end{array}$ \\
\hline Mollusca & Mollusca & 33 & A. Steuer & 1939 & XIX. - Mollusca & $\begin{array}{l}\text { http://ipt.medobis.eu/ } \\
\underline{\text { resource? }} \\
\underline{\text { r=egyptexpeditionmollu }} \\
\underline{\text { sca }}\end{array}$ \\
\hline $\begin{array}{l}\text { Tanaidacea, } \\
\text { Isopoda }\end{array}$ & $\begin{array}{l}\text { Tanaidacea, } \\
\text { Isopoda }\end{array}$ & 35 & H. J. Larwood & 1940 & $\begin{array}{l}\text { XXI. Tanaidacea } \\
\text { and Isopoda }\end{array}$ & $\begin{array}{l}\text { http://ipt.medobis.eu/ } \\
\text { resource? } \\
\underline{\text { r=egyptexpeditiontanaid }} \\
\underline{\text { aceaisopoda }}\end{array}$ \\
\hline
\end{tabular}

\section{Project description}

Title: Digitization of historical dataset - Egypt Expedition

This dataset combines the benthic macrofaunal data obtained from the floristical and faunistical survey of A. Steuer in the coasts near Alexandria in 1933. The results of this expedition were published in 38 volumes between 1934 and 1940. In this study, we present the digitized data of 14 volumes concerning macrofaunal taxa (1935-1940). In rare cases, 
occurrence data for planktonic species were available in these volumes and were included in the digitized datasets.

Personnel: The datasets were digitized by the LifeWatchGreece data management team. Irini Tsikopoulou (data manager), Stamatina Nikolopoulou (data, database and webgis application manager) and Aglaia Legaki (data manager) were the resource creators, Panagiotis D. Dimitriou (data manager) and Evangelia Avramidou (data manager) were content providers. Nicolas Bailly has checked difficult taxonomic cases.

The original data were collected by Dr. Adolf Steuer, professor at the University of Innsbruck, who organized and led the sampling expedition to the coasts near Alexandria, Egypt. After sampling, all collected specimens were preserved and sent to several experts for taxonomic identification. Each expert was responsible for the publication of his macrofaunal report.

Study area description: The study area of the Egypt Expedition is located between the Western and Eastern harbors of Alexandria, including nearby localities such as Abukir Bay, the Suez Canal and the lakes Edku and Mariout (Fig. 1). The majority of the sampling stations do not exceed the isobath of 200 meter. The coasts that were investigated were in part shallow and sandy, in part steep. Information concerning the sediment characteristics and vegetation of the studied area was also available and included in the digitized dataset.
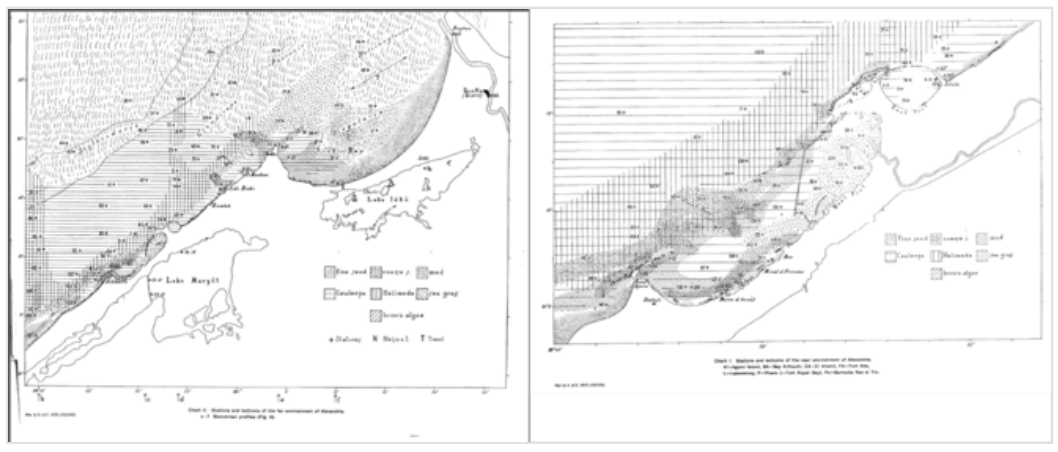

Figure 1.

Stations as they were mapped in Steuer's preliminary report (1935).

Design description: Data rescue/digitization

Funding: The digitization of this historical dataset was supported by the LifeWatchGreece Research Infrastructure, funded by the Greek Government under the General Secretariat of Research and Technology (GSRT), ESFRI Projects, Structural Funds, OPCE II.

Regarding the Egypt expedition, the Egyptian Ministry of Finance funded the whole sampling campaign and provided Dr Steuer with the expedition vehicle of the Marine Laboratory of Alexandria, a $15 \mathrm{~m}$ long motor boat named "El Hoot". Additionally, the Ministry of Finance provided access to the Marine Laboratory of Alexandria located within 
the harbor area of the Alexandria city, as well as a small row boat, an automobile and the sampling equipment.

\section{Sampling methods}

Sampling description: Sampling took place at 172 locations in the marine area off Alexandria, in the Suez Canal, in the Nile river and in two lagoons (Lake Mariout and Lake Edku). Adolf Steuer was in charge of the sampling which lasted from April to November of 1933. A motor-launch (small military vessel) $15 \mathrm{~m}$ long, named "El Hoot", belonging to the Marine Laboratory, was used for the one-day trips at sea. Since it was difficult to sail too far from the shore, only two stations (station 26 and station 64) surpassed the $200 \mathrm{~m}$ isobath. In some cases a small rowing boat was also used. The collection of benthic samples was done almost exclusively by using a dredge with an opening of $20 \times 70 \mathrm{~cm}$. In only one case sampling was performed with a large otter trawl (bottom trawling) in the eastern part of Bay of Abukir, at a depth of 20 meters. A bottom sampler (Petersen's grab) of $0.2 \mathrm{~m}^{2}$ surface was also used once in the Eastern harbor due to difficulties in its manipulation (Vatova 1935). In shallow water, where no other equipment could be used, the samples of benthos were taken by diving. The sites where the sampling was performed along the coast were: the mouth of the Nile near Rosetta (Rashid), Lake Mariout and Lake Edku. Concerning planktonic samples, vertical hauls were operated using a medium sized net with buckets of celluloid with a gauge bottom.

Quality control: Every single dataset was digitized manually from scanned documents. Some publications were in French or in German, depending on the author, and therefore the information was translated to English. Species names and sampling location names in the digitized datasets were kept same as in the original paper. Afterwards, all scientific names were cross-checked and taxonomically updated using the Taxon Match tool of the World Register of Marine Species (WoRMS) (WoRMS Editorial Board 2016). Station coordinates were produced by georeferencing maps from Fauvel (1937) using a Geographic Information System (GIS). The digitized datasets are presented in a standardised way, using Darwin Core terminology, informations on taxonomy, locality, sampling date, sampling protocol and individual measurements where they were available.

\section{Step description: Digitization process}

The digitization of the historical publications concerning the Egypt Expedition is a challenging process due to their complexity and the variety of the format across the different faunistic reports. Information on the sampling protocol and the sampling sites were digitized mainly based on the preliminary reports of Steuer (1935) and Vatova (1935) enriched with information from maps and the main text in the rest of the publications. 
Occurrence data were digitized based on the individual faunistic reports. The data digitization was made using the Darwin Core terminology.

The digitization process of the Egypt Expedition datasets included several steps that are described below:

1. Data managers read and comprehended individual faunistic publications, in order to overcome difficulties originating from the heterogeneity in the format and the content among the historical papers. The original authors did not follow a specific format for the presentation of their results. Some of them included species distribution maps, some reported species list, sampling dates and depths, while others also recorded individual species counts. If there was a species list in the historical papers, species were recorded according to their taxonomical classification. Respectively, if there was a station list, stations were reported chronologically.

2. A spreadsheet was created for each faunistic report and were populated with original species names found at each location. In this stage of digitization process, obvious typographic errors were corrected. The spreadsheets also contained information on the sampling depth (minimum and maximum depth), sampling date (year, month, day), sampling protocol and habitat (substrate type and vegetation). For benthic samples, station depth and sampling depth were matched. In some faunistic reports, station depths were given in fathoms (i.e. approximately 1.8 meters). In these cases, station depths in the datasets were converted to meters. For some taxonomic groups, additional information such as sex, lifestage, individual counts or body length measurements were available, either on a species level or on a specimen level. Accepted taxon names and taxonomic classification, as derived from the World Register of Marine species, were also included in the spreadsheets.

3. After the digitization of all available information contained in the main text and tables in the publications, sampling stations were georeferenced using the species distribution maps in every faunistic report. Since there were no stations coordinates, latitude, longitude and coordinates uncertainty were estimated using a GIS based on the distribution maps in each publication and in Fauvel (1937). In cases, where a station was only referred to as a specific locality in the text, and not accompanied by a symbol on a map, a new station with higher uncertainty was created based on the locality description.

4. In the next step, a code (fieldNumber) was created for each sampling event. A unique event was defined as a sampling event that took place in a specific station at a specific time and sampling depth using a specific sampling protocol. In some cases several samples had been taken in a location without defining the sampling station but only the wider area. To represent these, a new station ID was created, accompanied by an respective location remark. A code (occurrencelD) was also created for each species occurrence record.

5. The outcome of the above digitization steps was twelve spreadsheets with 56 columns containing occurrence data of twelve benthic macrofaunal taxa. These tables were combined in the MedOBIS PostgreSQL database in order to correct 
mistakes originating from differences in the information or absence of information derived from Steuer's preliminary report (1935) and individual faunistic reports. In cases of corrections, original information was always kept as a remark in the dataset.

6. The final step was the publication of the data through the MedOBIS Data Repository (MedOBIS IPT - http://ipt.medobis.eu/) and the MedOBIS Geoportal (htt p://medobis.portal.lifewatchgreece.eu/viewer). The Integrated Publishing Toolkit (IPT) (Wieczorek and Braak 2015, http://www.gbif.org/ipt) is a free open source software tool and is used to publish and share biodiversity datasets through the GBIF network (http://www.gbif.org/). It uses Darwin Core (http://rs.tdwg.org/dwc/ terms/) and Ecological Metadata Language (EML) standards. All datasets are distributed as Darwin Core Archives, a compressed file that contains: one or more data files with details for event, occurrence and measurements in a commaseparated or tab-separated list, an archive descriptor (meta.xml) file describing the individual data file columns used and a metadata file on EML describing the entire dataset. The datasets concerning different taxonomic groups were published separately with their metadata and individual URLs (Table 2). Since the repository does not currently support species measurements such as body and carapace length, this information is added in the supplementary files of the present paper (Suppl. material 1), following the recently developed Environmental data and Event schema of OBIS (De Pooter et al., in prep). The datasets are also available on MedOBIS Geoportal (a virtual lab on LifeWatchGreece Research Infrastructure), where all the taxonomic groups are visualized on a map. The Geoportal allows users to search and download marine species datasets from all over the Mediterranean in several different formats (CSV, KML, WFS, WMS). In addition, a MedOBIS mobile application has been developed on playstore (https:// play.google.com/store/apps/details?id=com.Hcmr.LifeWatch).

Table 2.

List of sampling stations, their locality, habitat characteristics and species richness

\begin{tabular}{|c|c|c|c|c|c|c|c|c|}
\hline$a / a$ & Station Name & Locality & Latitude & Longitude & $\begin{array}{l}\text { Coordi- } \\
\text { nates } \\
\text { uncer- } \\
\text { tainty }\end{array}$ & Habitat & $\begin{array}{l}\text { Number } \\
\text { of } \\
\text { species }\end{array}$ & $\begin{array}{l}\text { Number } \\
\text { of } \\
\text { phyla }\end{array}$ \\
\hline 1 & 1 & $\begin{array}{l}\text { North of Abu Qir } \\
\text { Bay }\end{array}$ & 31,363663 & 30,016892 & 200 & $\begin{array}{l}\text { mud and stones, } \\
\text { Halimeda }\end{array}$ & 19 & 4 \\
\hline 2 & 2 & $\begin{array}{l}\text { North of Pharo, } \\
\text { Alexandria }\end{array}$ & 31,288853 & 29,896681 & 200 & Halimeda, sand, mud & 35 & 4 \\
\hline 3 & 3 & $\begin{array}{l}\text { North of Pharo, } \\
\text { Alexandria }\end{array}$ & 31,344246 & 29,902678 & 200 & $\begin{array}{l}\text { muddy, Halimeda, } \\
\text { Caulerpa }\end{array}$ & 22 & 6 \\
\hline 4 & 4 & $\begin{array}{l}\text { Eastern Harbour, } \\
\text { Alexandria }\end{array}$ & 31,209188 & 29,884443 & 200 & sand, Caulerpa & 24 & 4 \\
\hline
\end{tabular}




\begin{tabular}{|c|c|c|c|c|c|c|c|c|}
\hline 5 & 5 & $\begin{array}{l}\text { Eastern Harbour, } \\
\text { Alexandria }\end{array}$ & 31,207894 & 29,890154 & 200 & $\begin{array}{l}\text { Caulerpa, Codium, } \\
\text { sand }\end{array}$ & 29 & 5 \\
\hline 6 & 6 & $\begin{array}{l}\text { Eastern Harbour, } \\
\text { Alexandria }\end{array}$ & 31,211206 & 29,901575 & 200 & stony, Caulerpa & 5 & 3 \\
\hline 7 & 7 & $\begin{array}{l}\text { Close to } \\
\text { Alexandria Harbour }\end{array}$ & 31,198758 & 29,826388 & 200 & $\begin{array}{l}\text { small stones, } \\
\text { Caulerpa }\end{array}$ & 38 & 5 \\
\hline 8 & 8 & $\begin{array}{l}\text { Close to } \\
\text { Alexandria Harbour }\end{array}$ & 31,189335 & 29,807257 & 200 & $\begin{array}{l}\text { small stones, } \\
\text { Caulerpa, Halimeda }\end{array}$ & 14 & 5 \\
\hline 9 & 9 & $\begin{array}{l}\text { Western Harbour, } \\
\text { Alexandria }\end{array}$ & 31,162977 & 29,854056 & 200 & mud with plants & 14 & 2 \\
\hline 10 & 10 & $\begin{array}{l}\text { Western Harbour, } \\
\text { Port-Quest, } \\
\text { Alexandria }\end{array}$ & 31,176319 & 29,855383 & 200 & Caulerpa, sand, mud & 16 & 4 \\
\hline 11 & 11 & $\begin{array}{l}\text { Western Harbour, } \\
\text { Alexandria }\end{array}$ & 31,191908 & 29,874419 & 200 & $\begin{array}{l}\text { smelling, black mud } \\
\text { without algae }\end{array}$ & 19 & 4 \\
\hline 12 & 12 & $\begin{array}{l}\text { Western Harbour, } \\
\text { Alexandria }\end{array}$ & 31,183076 & 29,853813 & 200 & $\begin{array}{l}\text { Caulerpa, Posidonia, } \\
\text { sand, some mud, } \\
\text { Codium ground }\end{array}$ & 14 & 3 \\
\hline 13 & 13 & $\begin{array}{l}\text { Western Harbour, } \\
\text { Alexandria }\end{array}$ & 31,18791 & 29,867852 & 200 & sand, some mud & 34 & 2 \\
\hline 14 & 14 & $\begin{array}{l}\text { Western Harbour, } \\
\text { Alexandria }\end{array}$ & 31,190708 & 29,859228 & 200 & $\begin{array}{l}\text { sandy, black mud } \\
\text { with little Caulerpa } \\
\text { and brown algae }\end{array}$ & 11 & 1 \\
\hline 15 & 15 & $\begin{array}{l}\text { Western Harbour, } \\
\text { Alexandria }\end{array}$ & 31,184171 & 29,862664 & 200 & sand & 8 & 2 \\
\hline 16 & 17 & $\begin{array}{l}\text { Western Harbour, } \\
\text { Port-Quest, } \\
\text { Alexandria }\end{array}$ & 31,168573 & 29,848745 & 200 & $\begin{array}{l}\text { little Caulerpa, sand, } \\
\text { black mud }\end{array}$ & 14 & 4 \\
\hline 17 & 18 & $\begin{array}{l}\text { Western Harbour, } \\
\text { Alexandria }\end{array}$ & 31,165604 & 29,848059 & 200 & $\begin{array}{l}\text { very little Caulerpa, } \\
\text { black mud }\end{array}$ & 5 & 1 \\
\hline 18 & 19 & $\begin{array}{l}\text { NW of Al Meks, } \\
\text { Egypt }\end{array}$ & 31,150471 & 29,835153 & 200 & Caulerpa, sand & 4 & 1 \\
\hline 19 & 20 & Off Al Meks, Egypt & 31,150471 & 29,835153 & 200 & $\begin{array}{l}\text { Posidonia, } \\
\text { Cymodocea }\end{array}$ & 6 & 3 \\
\hline 20 & 21 & $\begin{array}{l}\text { Near Dekheli, } \\
\text { Egypt }\end{array}$ & 31,137658 & 29,795965 & 200 & $\begin{array}{l}\text { Caulerpa, Posidonia, } \\
\text { Amphioxus, brown } \\
\text { algae }\end{array}$ & 22 & 6 \\
\hline
\end{tabular}




\begin{tabular}{|c|c|c|c|c|c|c|c|c|}
\hline 21 & 22 & $\begin{array}{l}\text { Great Pass, } \\
\text { Alexandria }\end{array}$ & 31,163205 & 29,82179 & 200 & $\begin{array}{l}\text { Posidonia, Caulerpa, } \\
\text { brown algae, rocks, } \\
\text { yellow sand }\end{array}$ & 19 & 4 \\
\hline 22 & 23 & $\begin{array}{l}\text { Margin of the } \\
\text { Great-Pass, } \\
\text { Alexandria }\end{array}$ & 31,169944 & 29,810483 & 200 & $\begin{array}{l}\text { yellow Amphioxus } \\
\text { sand, Halimeda, } \\
\text { Udotea }\end{array}$ & 10 & 5 \\
\hline 23 & 24 & Off Fort Ada & 31,219602 & 29,876927 & 200 & $\begin{array}{l}\text { stony, Caulerpa, } \\
\text { Halimeda }\end{array}$ & 8 & 4 \\
\hline 24 & 25 & $\begin{array}{l}\text { Infront of Fort Ada, } \\
\text { Alexandria }\end{array}$ & 31,207443 & 29,87276 & 350 & $\begin{array}{l}\text { Caulerpa, Halimeda, } \\
\text { brown algae, } \\
\text { seagrass }\end{array}$ & 11 & 4 \\
\hline 25 & 26 & $\begin{array}{l}\text { North of Al Max, } \\
\text { Alexandria }\end{array}$ & 31,386934 & 29,817017 & 200 & $\begin{array}{l}\text { soft and yellow mud } \\
\text { with Pteropodes }\end{array}$ & 11 & 3 \\
\hline 26 & 27 & $\begin{array}{l}\text { North of El Anfushi } \\
\text { Beach, Alexandria }\end{array}$ & 31,36923 & 29,862703 & 200 & $\begin{array}{l}\text { mud with mollusc } \\
\text { shells }\end{array}$ & 27 & 5 \\
\hline 27 & 28 & $\begin{array}{l}\text { Close to East } \\
\text { Harbour, } \\
\text { Alexandria }\end{array}$ & 31,227026 & 29,905195 & 200 & $\begin{array}{l}\text { stones, Caulerpa, } \\
\text { Halimeda }\end{array}$ & 37 & 5 \\
\hline 28 & 29 & $\begin{array}{l}\text { Close to East } \\
\text { Harbour, } \\
\text { Alexandria }\end{array}$ & 31,221752 & 29,908885 & 200 & stony, Caulerpa & 6 & 3 \\
\hline 29 & 30 & $\begin{array}{l}\text { Close to East } \\
\text { Harbour, } \\
\text { Alexandria }\end{array}$ & 31,219315 & 29,910256 & 200 & stones, Caulerpa & 14 & 6 \\
\hline 30 & 31 & $\begin{array}{l}\text { East Harbour, Port- } \\
\text { Quest, Alexandria }\end{array}$ & 31,203972 & 29,892629 & 200 & sand, Caulerpa & 14 & 3 \\
\hline 31 & 32 & $\begin{array}{l}\text { Eastern Harbour, } \\
\text { near entrance }\end{array}$ & 31,210863 & 29,89358 & 200 & $\begin{array}{l}\text { coarse sand with } \\
\text { stones and very little } \\
\text { mud, Caulerpa }\end{array}$ & 37 & 5 \\
\hline 32 & 35 & $\begin{array}{l}\text { Off Sidi Bishr, } \\
\text { Egypt }\end{array}$ & 31,280191 & 29,960736 & 200 & $\begin{array}{l}\text { Caulerpa, Posidonia, } \\
\text { Halimeda, rough } \\
\text { sand with stones, } \\
\text { Amphioxus, brown } \\
\text { algae }\end{array}$ & 51 & 6 \\
\hline 33 & 36 & Sidi Bischr & 31,263725 & 29,976631 & 200 & fine sand & 10 & 3 \\
\hline 34 & 37 & Sidi Bischr & 31,269151 & 29,971206 & 200 & sandy, Caulerpa & 4 & 2 \\
\hline 35 & 38 & NW of Abu Qir & 31,340535 & 30,002044 & 200 & $\begin{array}{l}\text { Halimeda, Caulerpa, } \\
\text { stony, brown algae }\end{array}$ & 8 & 5 \\
\hline
\end{tabular}




\begin{tabular}{|c|c|c|c|c|c|c|c|c|}
\hline 36 & 39 & NW of Abu Qir & 31,342819 & 30,025172 & 200 & $\begin{array}{l}\text { Caulerpa, sand, little } \\
\text { mud }\end{array}$ & 9 & 4 \\
\hline 37 & 40 & Abu Qir Bay & 31,383222 & 30,173793 & 200 & $\begin{array}{l}\text { sand, stones, } \\
\text { Amphioxus bottom }\end{array}$ & 11 & 4 \\
\hline 38 & 41 & $\begin{array}{l}\text { Crags at the } \\
\text { eastern coast of } \\
\text { Abu Qir Peninsula }\end{array}$ & 31,323736 & 30,073142 & 200 & $\begin{array}{l}\text { crags with } \\
\text { Cystoseira, Padina, } \\
\text { little Caulerpa }\end{array}$ & 15 & 4 \\
\hline 39 & 42 & $\begin{array}{l}\text { Abu Qir, eastern } \\
\text { coast }\end{array}$ & 31,307793 & 30,082184 & 200 & $\begin{array}{l}\text { gray sand, mud, sea } \\
\text { grass meadows }\end{array}$ & 15 & 4 \\
\hline 40 & 43 & $\begin{array}{l}\text { Western side of } \\
\text { Abu Qir }\end{array}$ & 31,326115 & 30,059817 & 200 & $\begin{array}{l}\text { crags, sand, brown } \\
\text { algae, Posidonia, } \\
\text { Caulerpa }\end{array}$ & 8 & 3 \\
\hline 41 & 44 & Abu Qir Bay & 31,473356 & 30,208724 & 200 & fine rich slime & 4 & 3 \\
\hline 42 & 45 & Abu Qir Bay & 31,433191 & 30,2171 & 200 & little sand, much mud & 7 & 5 \\
\hline 43 & 47 & Abu Qir Bay & 31,34753 & 30,232328 & 200 & sandy with little mud & 7 & 3 \\
\hline 44 & 48 & $\begin{array}{l}\text { Lake Edku, } \\
\text { seaside, sandy } \\
\text { beach near the } \\
\text { channel, in open } \\
\text { water and upon } \\
\text { stones of the } \\
\text { bridge }\end{array}$ & 31,273053 & 30,166655 & 200 & shallow sand & 27 & 3 \\
\hline 45 & 49 & $\begin{array}{l}\text { Lake Edku, at the } \\
\text { Bridge, near the } \\
\text { canal with the Bay } \\
\text { of Abu Qir, on } \\
\text { shallow see-weed- } \\
\text { bottom of the lake }\end{array}$ & 31,254493 & 30,202109 & 800 & $\begin{array}{l}\text { shallow seagrass } \\
\text { meadows }\end{array}$ & 9 & 3 \\
\hline 46 & 50 & Off Abu Qir & 31,359903 & 30,07219 & 200 & $\begin{array}{l}\text { sandy, stony, } \\
\text { Amphioxus bottom }\end{array}$ & 20 & 5 \\
\hline 47 & 51 & Abu Qir Bay & 31,401544 & 30,082422 & 200 & $\begin{array}{l}\text { sandy ground, } \\
\text { Amphioxus ground }\end{array}$ & 18 & 4 \\
\hline 48 & 52 & North of Abu Qir & 31,459032 & 30,065433 & 200 & $\begin{array}{l}\text { mud, Phoronis } \\
\text { bottom }\end{array}$ & 24 & 5 \\
\hline 49 & 53 & $\begin{array}{l}\text { Off Abu Qir- } \\
\text { Montazah }\end{array}$ & 31,413346 & 29,992907 & 200 & $\begin{array}{l}\text { yellow mud, bottom } \\
\text { with Enteropneustes }\end{array}$ & 24 & 5 \\
\hline 50 & 54 & $\begin{array}{l}\text { Northwest of Abu } \\
\text { Qir Bay }\end{array}$ & 31,456747 & 29,955787 & 200 & & 17 & 4 \\
\hline
\end{tabular}




\begin{tabular}{|c|c|c|c|c|c|c|c|c|}
\hline 51 & 55 & $\begin{array}{l}\text { Northwest of Abu } \\
\text { Qir Bay }\end{array}$ & 31,396785 & 29,963211 & 200 & mud & 25 & 4 \\
\hline 52 & 56 & Abu Qir Bay & 31,343009 & 30,128584 & 200 & $\begin{array}{l}\text { coarse Amphioxus } \\
\text { sand, little Caulerpa }\end{array}$ & 9 & 4 \\
\hline 53 & 57 & Abu Qir Bay & 31,331588 & 30,13715 & 200 & fine sand, Caulerpa & 21 & 3 \\
\hline 54 & 58 & Abu Qir Bay & 31,307793 & 30,165227 & 200 & $\begin{array}{l}\text { sandy ground with } \\
\text { algae (Caulerpa) and } \\
\text { seaweed } \\
\text { (Cymodocea) }\end{array}$ & 23 & 4 \\
\hline 55 & 60 & North of Sidi Bishr & 31,363948 & 29,950933 & 200 & $\begin{array}{l}\text { mud, bottom with } \\
\text { Enteropneustes }\end{array}$ & 8 & 4 \\
\hline 56 & 61 & $\begin{array}{l}\text { North of East Port } \\
\text { of Alexandria }\end{array}$ & 31,390218 & 29,906104 & 200 & $\begin{array}{l}\text { stones, mud, algae } \\
\text { (one empty shell) }\end{array}$ & 60 & 5 \\
\hline 57 & 62 & $\begin{array}{l}\text { North of Pharo, } \\
\text { Alexandria }\end{array}$ & 31,323117 & 29,931517 & 200 & $\begin{array}{l}\text { mud, Caulerpa } \\
\text { bottom }\end{array}$ & 27 & 5 \\
\hline 58 & 63 & $\begin{array}{l}\text { NW of Agami } \\
\text { island }\end{array}$ & 31,313848 & 29,722266 & 200 & $\begin{array}{l}\text { stony coarse sand } \\
\text { and mud }\end{array}$ & 31 & 5 \\
\hline 59 & 64 & $\begin{array}{l}\text { NW of Agami } \\
\text { island }\end{array}$ & 31,325667 & 29,710205 & 200 & $\begin{array}{l}\text { muddy ground mixed } \\
\text { with coarse sand }\end{array}$ & 23 & 6 \\
\hline 60 & 65 & North of Agamy & 31,267858 & 29,790125 & 200 & $\begin{array}{l}\text { sand, mud, little } \\
\text { Caulerpa }\end{array}$ & 1 & 1 \\
\hline 61 & 66 & $\begin{array}{l}\text { North of West Port } \\
\text { of Alexandria }\end{array}$ & 31,238162 & 29,853228 & 200 & sand, mud, Caulerpa & 18 & 3 \\
\hline 62 & 67 & $\begin{array}{l}\text { North of West Port } \\
\text { of Alexandria }\end{array}$ & 31,274425 & 29,845804 & 200 & sand, mud & 22 & 4 \\
\hline 63 & 68 & $\begin{array}{l}\text { North of West Port } \\
\text { of Alexandria }\end{array}$ & 31,328684 & 29,84538 & 200 & Caulerpa, mud & 14 & 4 \\
\hline 64 & 69 & $\begin{array}{l}\text { North of West Port } \\
\text { of Alexandria }\end{array}$ & 31,345125 & 29,842823 & 200 & Caulerpa, mud & 15 & 4 \\
\hline 65 & 71 & $\begin{array}{l}\text { North of East Port } \\
\text { of Alexandria }\end{array}$ & 31,275718 & 29,900393 & 200 & $\begin{array}{l}\text { stony, Caulerpa, } \\
\text { Halimeda }\end{array}$ & 2 & 2 \\
\hline 66 & 72 & $\begin{array}{l}\text { North of Pharo, } \\
\text { Alexandria }\end{array}$ & 31,317692 & 29,894112 & 200 & $\begin{array}{l}\text { muddy, sandy, } \\
\text { Caulerpa }\end{array}$ & 16 & 2 \\
\hline 67 & 73 & $\begin{array}{l}\text { North of East Port } \\
\text { of Alexandria }\end{array}$ & 31,352527 & 29,890114 & 200 & $\begin{array}{l}\text { Caulerpa, Halimeda, } \\
\text { mud }\end{array}$ & 13 & 5 \\
\hline
\end{tabular}




\begin{tabular}{|c|c|c|c|c|c|c|c|c|}
\hline 68 & 74 & North of Sidi Bishr & 31,32226 & 29,964924 & 200 & $\begin{array}{l}\text { mud, Caulerpa, } \\
\text { bottom with } \\
\text { Enteropneustes }\end{array}$ & 18 & 6 \\
\hline 69 & 75 & $\begin{array}{l}\text { North of Pharo, } \\
\text { Alexandria }\end{array}$ & 31,305289 & 29,932008 & 200 & sand, mud & 12 & 4 \\
\hline 70 & 76 & $\begin{array}{l}\text { Infront of East } \\
\text { Harbour, } \\
\text { Alexandria }\end{array}$ & 31,220876 & 29,891905 & 200 & $\begin{array}{l}\text { stony, sandy, } \\
\text { Caulerpa, Halimeda }\end{array}$ & 15 & 4 \\
\hline 71 & 77 & $\begin{array}{l}\text { North of Eastern } \\
\text { Harbour, } \\
\text { Alexandria }\end{array}$ & 31,227577 & 29,884024 & 200 & $\begin{array}{l}\text { Caulerpa, stones, } \\
\text { Halimeda }\end{array}$ & 18 & 6 \\
\hline 72 & 78 & $\begin{array}{l}\text { Close to West } \\
\text { Harbour of } \\
\text { Alexandria }\end{array}$ & 31,202031 & 29,856423 & 200 & $\begin{array}{l}\text { Caulerpa, Halimeda, } \\
\text { Posidonia, stony, } \\
\text { brown algae }\end{array}$ & 29 & 6 \\
\hline 73 & 79 & $\begin{array}{l}\text { Close to West } \\
\text { Harbour of } \\
\text { Alexandria }\end{array}$ & 31,20761 & 29,850944 & 200 & $\begin{array}{l}\text { Caulerpa, Halimeda, } \\
\text { stony, rough sand }\end{array}$ & 3 & 3 \\
\hline 74 & 80 & $\begin{array}{l}\text { North-West of } \\
\text { Harbour, } \\
\text { Alexandria }\end{array}$ & 31,211893 & 29,845233 & 200 & $\begin{array}{l}\text { sandy, stony, } \\
\text { Caulerpa, Halimeda }\end{array}$ & 1 & 1 \\
\hline 75 & 81 & $\begin{array}{l}\text { Outer side of Fort } \\
\text { El-Ayana }\end{array}$ & 31,150983 & 29,784888 & 200 & $\begin{array}{l}\text { rocky Amphioxus } \\
\text { sand, Posidonia }\end{array}$ & 3 & 2 \\
\hline 76 & 82 & $\begin{array}{l}\text { Outer side of Fort } \\
\text { El-Ayana }\end{array}$ & 31,146723 & 29,783032 & 200 & $\begin{array}{l}\text { Halimeda and brown } \\
\text { algae }\end{array}$ & 4 & 3 \\
\hline 77 & 83 & $\begin{array}{l}\text { Inner side of the } \\
\text { Fort el Ayana }\end{array}$ & 31,14451 & 29,785435 & 200 & $\begin{array}{l}\text { crags with Padina } \\
\text { pav., shallow } \\
\text { Posidonia bottom }\end{array}$ & 6 & 2 \\
\hline 78 & 84 & $\begin{array}{l}\text { Isle Agami, inside } \\
\text { shoal beach }\end{array}$ & 31,14708 & 29,789195 & 200 & $\begin{array}{l}\text { Posidonia, little } \\
\text { Caulerpa, shoall } \\
\text { beach with stones, } \\
\text { brown algae }\end{array}$ & 2 & 2 \\
\hline 79 & 85 & Isle Agami, inside & 31,149531 & 29,796785 & 200 & Caulerpa, Posidonia & 4 & 3 \\
\hline 80 & 86 & Isle Agami, outside & 31,154137 & 29,803791 & 200 & $\begin{array}{l}\text { coarse sand, } \\
\text { Amphioxus, } \\
\text { Posidonia, Caulerpa }\end{array}$ & 4 & 4 \\
\hline 81 & 87 & $\begin{array}{l}\text { Close to El } \\
\text { Dukhaylah Beach, } \\
\text { Egypt }\end{array}$ & 31,146876 & 29,811992 & 200 & Caulerpa, gray sand & 3 & 2 \\
\hline 82 & 90 & $\begin{array}{l}\text { North of Agami } \\
\text { island }\end{array}$ & 31,167349 & 29,78213 & 200 & Caulerpa, mud & 12 & 3 \\
\hline
\end{tabular}




\begin{tabular}{|c|c|c|c|c|c|c|c|c|}
\hline 83 & 91 & $\begin{array}{l}\text { North of Agami } \\
\text { island }\end{array}$ & 31,198758 & 29,778132 & 200 & $\begin{array}{l}\text { muddy, Caulerpa, } \\
\text { Cymodocea }\end{array}$ & 7 & 2 \\
\hline 84 & 92 & $\begin{array}{l}\text { North of Agami } \\
\text { island }\end{array}$ & 31,238162 & 29,778132 & 200 & Caulerpa, mud & 10 & 3 \\
\hline 85 & 93 & $\begin{array}{l}\text { North of Port of El- } \\
\text { Dekheila }\end{array}$ & 31,159281 & 29,794416 & 200 & $\begin{array}{l}\text { coarse Amphioxus } \\
\text { sand, Caulerpa, } \\
\text { Halimeda, Posidonia }\end{array}$ & 3 & 3 \\
\hline 86 & 94 & $\begin{array}{l}\text { North of Port of El- } \\
\text { Dekheila }\end{array}$ & 31,160433 & 29,801688 & 200 & $\begin{array}{l}\text { stony, Caulerpa, } \\
\text { Halimeda, } \\
\text { Sargassum, } \\
\text { Posidonia }\end{array}$ & 7 & 4 \\
\hline 87 & 95 & $\begin{array}{l}\text { North of Port of El- } \\
\text { Dekheila }\end{array}$ & 31,160486 & 29,806092 & 200 & $\begin{array}{l}\text { stony, Caulerpa, } \\
\text { Halimeda, } \\
\text { Sargassum, } \\
\text { Posidonia }\end{array}$ & 3 & 1 \\
\hline 88 & 97 & $\begin{array}{l}\text { close to Port of El- } \\
\text { Dekheila }\end{array}$ & 31,159765 & 29,822968 & 200 & $\begin{array}{l}\text { Caulerpa, Halimeda, } \\
\text { Posidonia, stony, } \\
\text { Amphioxus bottom, } \\
\text { brown algae }\end{array}$ & 3 & 3 \\
\hline 89 & 98 & $\begin{array}{l}\text { West of the } \\
\text { Western Harbour } \\
\text { of Alexandria }\end{array}$ & 31,174891 & 29,840631 & 200 & $\begin{array}{l}\text { fine sand, Posidonia, } \\
\text { Caulerpa }\end{array}$ & 8 & 3 \\
\hline 90 & 99 & $\begin{array}{l}\text { Close to Western } \\
\text { Harbour of } \\
\text { Alexandria }\end{array}$ & 31,187071 & 29,841725 & 200 & $\begin{array}{l}\text { stones, sand, } \\
\text { Posidonia, Caulerpa, } \\
\text { Halimeda }\end{array}$ & 6 & 3 \\
\hline 91 & 100 & $\begin{array}{l}\text { West of the } \\
\text { Western Harbour } \\
\text { of Alexandria }\end{array}$ & 31,178453 & 29,821961 & 200 & $\begin{array}{l}\text { Caulerpa, Halimeda, } \\
\text { mussel-sand }\end{array}$ & 9 & 2 \\
\hline 92 & 101 & Near Agami island & 31,141831 & 29,772552 & 200 & $\begin{array}{l}\text { Caulerpa, Posidonia, } \\
\text { Halimeda, brown } \\
\text { algae, sand }\end{array}$ & 7 & 3 \\
\hline 93 & 102 & $\begin{array}{l}\text { Close to Agamy } \\
\text { Beach }\end{array}$ & 31,099868 & 29,725917 & 200 & $\begin{array}{l}\text { Cystoseira (brown } \\
\text { algae), Caulerpa- } \\
\text { Halimeda (green } \\
\text { algae), stonny }\end{array}$ & 18 & 3 \\
\hline 94 & 103 & $\begin{array}{l}\text { North of Agamy } \\
\text { Beach }\end{array}$ & 31,14127 & 29,728202 & 200 & Caulerpa, sand, mud & 12 & 2 \\
\hline 95 & 104 & $\begin{array}{l}\text { North of Agamy } \\
\text { Beach }\end{array}$ & 31,123853 & 29,726488 & 200 & $\begin{array}{l}\text { coarse sand, } \\
\text { Amphioxus sand, } \\
\text { Halimeda, Caulerpa }\end{array}$ & 4 & 3 \\
\hline
\end{tabular}




\begin{tabular}{|c|c|c|c|c|c|c|c|c|}
\hline 96 & 105 & $\begin{array}{l}\text { Close to Abu Talat, } \\
\text { Egypt }\end{array}$ & 31,053897 & 29,660244 & 200 & $\begin{array}{l}\text { dark sand, rotten, } \\
\text { Posidonia with } \\
\text { Cystoseira }\end{array}$ & 11 & 3 \\
\hline 97 & 106 & $\begin{array}{l}\text { Close to Abu Talat, } \\
\text { Egypt }\end{array}$ & 31,08245 & 29,658531 & 200 & coarse sand & 1 & 1 \\
\hline 98 & 108 & $\begin{array}{l}\text { North of Abu Talat, } \\
\text { Egypt }\end{array}$ & 31,117571 & 29,660244 & 200 & $\begin{array}{l}\text { Halimeda, Caulerpa, } \\
\text { brown algae, sand, } \\
\text { stones }\end{array}$ & 5 & 3 \\
\hline 99 & 109 & $\begin{array}{l}\text { North of Abu Talat, } \\
\text { Egypt }\end{array}$ & 31,149836 & 29,665955 & 200 & $\begin{array}{l}\text { sand, stones, } \\
\text { Dasycladus, } \\
\text { Halimeda, Cystoseira }\end{array}$ & 2 & 2 \\
\hline 100 & 110 & Near Agami island & 31,134132 & 29,770613 & 200 & $\begin{array}{l}\text { crags with shallow } \\
\text { sand bottom }\end{array}$ & 2 & 1 \\
\hline 101 & 111 & Near Agami island & 31,143174 & 29,76633 & 200 & $\begin{array}{l}\text { stony, Caulerpa, } \\
\text { Halimeda, Posidonia }\end{array}$ & 10 & 5 \\
\hline 102 & 112 & Near Agami island & 31,149361 & 29,763475 & 200 & $\begin{array}{l}\text { Caulerpa, Halimeda, } \\
\text { finer sand, little mud, } \\
\text { Amphioxus bottom }\end{array}$ & 7 & 4 \\
\hline 103 & 113 & Near Agami island & 31,174488 & 29,733589 & 200 & Caulerpa, sand, mud & 3 & 3 \\
\hline 104 & 114 & $\begin{array}{l}\text { North of Abu Talat, } \\
\text { Egypt }\end{array}$ & 31,176106 & 29,672808 & 200 & $\begin{array}{l}\text { stony, sand, mud, } \\
\text { Caulerpa, Halimeda }\end{array}$ & 20 & 4 \\
\hline 105 & 115 & $\begin{array}{l}\text { North of Abu Talat, } \\
\text { Egypt }\end{array}$ & 31,19438 & 29,666526 & 200 & $\begin{array}{l}\text { stony, Caulerpa, } \\
\text { Halimeda }\end{array}$ & 7 & 4 \\
\hline 106 & 116 & $\begin{array}{l}\text { North of Abu Talat, } \\
\text { Egypt }\end{array}$ & 31,219888 & 29,659635 & 200 & $\begin{array}{l}\text { Caulerpa, Halimeda, } \\
\text { sand, mud, brown } \\
\text { algae }\end{array}$ & 12 & 5 \\
\hline 107 & 117 & $\begin{array}{l}\text { North of Abu Talat, } \\
\text { Egypt }\end{array}$ & 31,257007 & 29,660777 & 200 & $\begin{array}{l}\text { mud, stones, } \\
\text { Caulerpa, Halimeda } \\
\text { sitting on calcareus } \\
\text { algae }\end{array}$ & 3 & 3 \\
\hline 108 & 119 & $\begin{array}{l}\text { Great Pass, } \\
\text { Alexandria }\end{array}$ & 31,170344 & 29,820762 & 200 & $\begin{array}{l}\text { yellow sand, stones, } \\
\text { mud, Amphioxus } \\
\text { sand, Caulerpa, } \\
\text { Posidonia }\end{array}$ & 23 & 5 \\
\hline 109 & 121 & $\begin{array}{l}\text { West of the } \\
\text { Western Harbour } \\
\text { of Alexandria }\end{array}$ & 31,176454 & 29,833154 & 200 & $\begin{array}{l}\text { yellow coarse sand, } \\
\text { stony, Amphioxus } \\
\text { sand, Caulerpa, } \\
\text { Halimeda }\end{array}$ & 7 & 4 \\
\hline
\end{tabular}




\begin{tabular}{|c|c|c|c|c|c|c|c|c|}
\hline 110 & 122 & $\begin{array}{l}\text { West of the } \\
\text { Western Harbour } \\
\text { of Alexandria }\end{array}$ & 31,173256 & 29,827215 & 200 & $\begin{array}{l}\text { coarse sand, } \\
\text { Caulerpa, Amphioxus }\end{array}$ & 4 & 4 \\
\hline 111 & 124 & $\begin{array}{l}\text { Close to Western } \\
\text { Harbour of } \\
\text { Alexandria }\end{array}$ & 31,167546 & 29,841435 & 200 & fine sand, Caulerpa & 1 & 1 \\
\hline 112 & 125 & $\begin{array}{l}\text { West of the } \\
\text { Western Harbour } \\
\text { of Alexandria }\end{array}$ & 31,166974 & 29,825788 & 200 & $\begin{array}{l}\text { Halimeda, Caulerpa, } \\
\text { yellow sand, stones, } \\
\text { brown algae, } \\
\text { Amphioxus bottom }\end{array}$ & 32 & 5 \\
\hline 113 & 126 & $\begin{array}{l}\text { West of the } \\
\text { western Harbour of } \\
\text { Alexandria }\end{array}$ & 31,162749 & 29,832755 & 200 & $\begin{array}{l}\text { Caulerpa, Posidonia, } \\
\text { dark sand }\end{array}$ & 1 & 1 \\
\hline 114 & 128 & $\begin{array}{l}\text { Port Al-Dikheila, } \\
\text { Egypt }\end{array}$ & 31,140061 & 29,803234 & 200 & $\begin{array}{l}\text { sand, mud, Caulerpa, } \\
\text { Posidonia }\end{array}$ & 2 & 2 \\
\hline 115 & 134 & $\begin{array}{l}\text { East of Dekheila } \\
\text { Port }\end{array}$ & 31,148857 & 29,827965 & 200 & $\begin{array}{l}\text { Posidonia, Caulerpa, } \\
\text { rough sand, little } \\
\text { mud, Amphioxus } \\
\text { bottom }\end{array}$ & 8 & 3 \\
\hline 116 & 135 & $\begin{array}{l}\text { Close to El } \\
\text { Dukhaylah Beach }\end{array}$ & 31,142015 & 29,825911 & 200 & $\begin{array}{l}\text { stony, sandy, } \\
\text { Caulerpa, Halimeda, } \\
\text { Posidonia }\end{array}$ & 13 & 4 \\
\hline 117 & 136 & $\begin{array}{l}\text { Close to El } \\
\text { Dukhaylah Beach }\end{array}$ & 31,14311 & 29,82553 & 200 & $\begin{array}{l}\text { Caulerpa, Posidonia, } \\
\text { Amphioxus bottom }\end{array}$ & 10 & 4 \\
\hline 118 & 137 & $\begin{array}{l}\text { Close to El } \\
\text { Dukhaylah Beach }\end{array}$ & 31,139371 & 29,818676 & 200 & $\begin{array}{l}\text { dark sandy bottom, } \\
\text { Caulerpa, little brown } \\
\text { algae, Cymodocea }\end{array}$ & 4 & 2 \\
\hline 119 & 138 & $\begin{array}{l}\text { Close to El } \\
\text { Dukhaylah Beach }\end{array}$ & 31,140703 & 29,819652 & 200 & $\begin{array}{l}\text { Caulerpa, brown- } \\
\text { algae, Posidonia, } \\
\text { Amphioxus bottom }\end{array}$ & 3 & 2 \\
\hline 120 & 139 & $\begin{array}{l}\text { NW of Al Meks, } \\
\text { Egypt }\end{array}$ & 31,148629 & 29,832619 & 200 & $\begin{array}{l}\text { Caulerpa, Posidonia, } \\
\text { rough sand }\end{array}$ & 5 & 2 \\
\hline 121 & 140 & $\begin{array}{l}\text { Entrance of } \\
\text { Western Harbour, } \\
\text { Alexandria }\end{array}$ & 31,156752 & 29,841035 & 200 & $\begin{array}{l}\text { Caulerpa, Posidonia, } \\
\text { stones, Amphioxus } \\
\text { bottom, rough sand }\end{array}$ & 19 & 5 \\
\hline 122 & 141 & $\begin{array}{l}\text { Western Harbour, } \\
\text { Port-Quest, } \\
\text { Alexandria }\end{array}$ & 31,173987 & 29,857049 & 200 & sand, black mud & 3 & 2 \\
\hline 123 & 142 & NW of AI Max & 31,154798 & 29,831545 & 200 & mud & 1 & 1 \\
\hline
\end{tabular}




\begin{tabular}{|c|c|c|c|c|c|c|c|c|}
\hline 124 & 143 & $\begin{array}{l}\text { North of Port of El- } \\
\text { Dekheila }\end{array}$ & 31,170407 & 29,799984 & 200 & $\begin{array}{l}\text { Halimeda, stony, } \\
\text { Amphioxus bottom }\end{array}$ & 6 & 3 \\
\hline 125 & 144 & $\begin{array}{l}\text { North of Agami } \\
\text { island }\end{array}$ & 31,174202 & 29,791552 & 200 & $\begin{array}{l}\text { Caulerpa, Halimeda, } \\
\text { rough sand, mud }\end{array}$ & 9 & 4 \\
\hline 126 & 145 & $\begin{array}{l}\text { NW of West Port of } \\
\text { Alexandria }\end{array}$ & 31,219734 & 29,833656 & 200 & $\begin{array}{l}\text { light gray mud, little } \\
\text { Caulerpa }\end{array}$ & 7 & 4 \\
\hline 127 & 146 & $\begin{array}{l}\text { Off the Eastern } \\
\text { Harbour, } \\
\text { Alexandria }\end{array}$ & 31,216802 & 29,89358 & 200 & $\begin{array}{l}\text { Caulerpa, Halimeda, } \\
\text { Posidonia, } \\
\text { Dasycladus }\end{array}$ & 15 & 5 \\
\hline 128 & 147 & Nile mouth & 31,453993 & 30,375344 & 2000 & mud & 8 & 2 \\
\hline 129 & 148 & Off Rosetta & 31,397124 & 30,426265 & 1000 & sandy & 1 & 1 \\
\hline 130 & $12 I X$ & $\begin{array}{l}\text { Lake Mareotis, } \\
\text { near the Mex } \\
\text { Experimental } \\
\text { station or in open } \\
\text { water }\end{array}$ & 31,134285 & 29,855493 & 600 & mud & 4 & 2 \\
\hline 131 & $13 d$ & $\begin{array}{l}\text { Western Harbour, } \\
\text { arsenal basin }\end{array}$ & 31,192419 & 29,863142 & 500 & muddy, sand & 1 & 1 \\
\hline 132 & $14 I X$ & $\begin{array}{l}\text { Lake Mareotis, } \\
\text { eastward, near the } \\
\text { fresh-water fish- } \\
\text { market, at the } \\
\text { coast and towards } \\
\text { the middle of the } \\
\text { lake }\end{array}$ & 31,168891 & 29,906109 & 1000 & mud & 4 & 2 \\
\hline 133 & $25 a$ & $\begin{array}{l}\text { Infront of Fort Ada, } \\
\text { Alexandria }\end{array}$ & 31,209214 & 29,870289 & 200 & $\begin{array}{l}\text { Amphioxus sand, } \\
\text { Caulerpa, } \\
\text { Cymodocea }\end{array}$ & 2 & 2 \\
\hline 134 & $25 c$ & $\begin{array}{l}\text { Infront of Fort Ada, } \\
\text { Alexandria }\end{array}$ & 31,206832 & 29,873395 & 200 & $\begin{array}{l}\text { sand with Cystoseira, } \\
\text { red algae, Caulerpa, } \\
\text { Ulva }\end{array}$ & 5 & 2 \\
\hline 135 & $25 d$ & $\begin{array}{l}\text { Infront of Fort Ada, } \\
\text { Alexandria }\end{array}$ & 31,206085 & 29,874797 & 200 & $\begin{array}{l}\text { near the land sandy } \\
\text { seagrass meadows, } \\
\text { Ulva }\end{array}$ & 4 & 2 \\
\hline
\end{tabular}




\begin{tabular}{|c|c|c|c|c|c|c|c|c|}
\hline 136 & $28 I X$ & $\begin{array}{l}\text { Lake Mareotis, on } \\
\text { the mole extending } \\
\text { from the Mex } \\
\text { Experimental } \\
\text { station to the } \\
\text { south, on floating } \\
\text { sea weed and in } \\
\text { open water and on } \\
\text { stalks of reed in } \\
\text { mud }\end{array}$ & 31,113726 & 29,858768 & 1000 & mud & 12 & 4 \\
\hline 137 & $33 a$ & $\begin{array}{l}\text { Head of the pier of } \\
\text { Silsila, Alexandria }\end{array}$ & 31,215089 & 29,904621 & 200 & Caulerpa & 4 & 3 \\
\hline 138 & $33 b$ & Off Silsila & 31,212615 & 29,907514 & 200 & $\begin{array}{l}\text { sandy ground with } \\
\text { Cypraca }\end{array}$ & 9 & 3 \\
\hline 139 & $34 a$ & $\begin{array}{l}\text { East Harbour, } \\
\text { Silsila corner }\end{array}$ & 31,20934 & 29,905116 & 200 & $\begin{array}{l}\text { shallow sandy, } \\
\text { Codium, Caulerpa }\end{array}$ & 18 & 4 \\
\hline 140 & $34 b$ & $\begin{array}{l}\text { East Harbour, off } \\
\text { Silsila }\end{array}$ & 31,211206 & 29,903745 & 200 & sand with Caulerpa & 5 & 4 \\
\hline 141 & $59 a$ & North of Sidi Bishr & 31,307127 & 29,977202 & 200 & $\begin{array}{l}\text { coarse sand, } \\
\text { begrown with } \\
\text { Caulerpa and } \\
\text { Halimeda }\end{array}$ & 6 & 3 \\
\hline 142 & $59 b$ & North of Sidi Bishr & 31,299703 & 29,987196 & 200 & $\begin{array}{l}\text { coarse Amphioxus } \\
\text { sand with algae }\end{array}$ & 12 & 5 \\
\hline 143 & aquarium & $\begin{array}{l}\text { Aquarium of } \\
\text { Laboratory, } \\
\text { Alexandria }\end{array}$ & 31,212715 & 29,884665 & 200 & & 2 & 1 \\
\hline 144 & BA & $\begin{array}{l}\text { Off the Barracks of } \\
\text { Ras el Tin, Po, } \\
\text { Anfouchi Bay }\end{array}$ & 31,205991 & 29,870975 & 200 & $\begin{array}{l}\text { Foraminifera sand in } \\
\text { Posidonia meadows }\end{array}$ & 1 & 1 \\
\hline 145 & $\begin{array}{l}\text { coastAbuQir-near } \\
\text { Rosetta }\end{array}$ & $\begin{array}{l}\text { At the coast of the } \\
\text { Bay of Abu Qir, } \\
\text { washed ashore } \\
\text { near Rosette }\end{array}$ & 31,412646 & 30,34421 & 200 & sand & 8 & 3 \\
\hline 146 & D1 & $\begin{array}{l}\text { Lake Edku, near } \\
\text { isle Derfil }\end{array}$ & 31,269663 & 30,254223 & 200 & black mud & 10 & 3 \\
\hline 147 & D2 & $\begin{array}{l}\text { Lake Edku, near } \\
\text { the village }\end{array}$ & 31,294851 & 30,309155 & 600 & mud, stones, sand & 8 & 2 \\
\hline
\end{tabular}




\begin{tabular}{|c|c|c|c|c|c|c|c|c|}
\hline 148 & eastharbour1 & $\begin{array}{l}\text { Eastern Harbour: } \\
\text { under the hull of a } \\
\text { ship or epifauna or } \\
\text { on the body of a } \\
\text { ship or infront of } \\
\text { bath and } \\
\text { laboratory or } \\
\text { Kayed Bay }\end{array}$ & 31,208182 & 29,894361 & 1300 & $\begin{array}{l}\text { Ulva and coralline } \\
\text { zone, Caulerpa }\end{array}$ & 31 & 6 \\
\hline 149 & FortAda & Fort Ada & 31,210875 & 29,875577 & 200 & crags & 5 & 1 \\
\hline 150 & L & $\begin{array}{l}\text { Eastern Harbour, } \\
\text { off the } \\
\text { Laboratory,near } \\
\text { the laboratory, } \\
\text { before the } \\
\text { laboratory }\end{array}$ & 31,211614 & 29,884181 & 200 & $\begin{array}{l}\text { Ulva-coralline zone, } \\
\text { rocks, Caulerpa }\end{array}$ & 17 & 3 \\
\hline 151 & LacMarioutcenter & $\begin{array}{l}\text { Lake Mayrut, in the } \\
\text { middle of the lake, } \\
\text { mud between the } \\
\text { reeds on the bank }\end{array}$ & 31,153046 & 29,898617 & 6000 & mud & 2 & 1 \\
\hline 152 & $\begin{array}{l}\text { LakeEdku_marine } \\
\text { beach }\end{array}$ & $\begin{array}{l}\text { Lake Edku,marine } \\
\text { beach }\end{array}$ & 31,271933 & 30,17621 & 200 & sandy beach & 1 & 1 \\
\hline 153 & LakeEdkubridge & $\begin{array}{l}\text { Lake Edku, bridge, } \\
\text { under the stones } \\
\text { and among sea } \\
\text { weeds, near Edku } \\
\text { Channel }\end{array}$ & 31,267557 & 30,179252 & 400 & mud, stones & 4 & 1 \\
\hline 154 & nearbath & $\begin{array}{l}\text { Eastern Harbour, } \\
\text { near the bath, } \\
\text { Alexandria }\end{array}$ & 31,212364 & 29,885371 & 200 & $\begin{array}{l}\text { Caulerpa, stones, } \\
\text { sand }\end{array}$ & 24 & 4 \\
\hline 155 & offSidiBishr & Off Sidi Bishr & 31,271107 & 29,98531 & 200 & & 1 & 1 \\
\hline 156 & Pharo & $\begin{array}{l}\text { Pharo, outerside or } \\
\text { Kayed Bay outside } \\
\text { in Kalkagen }\end{array}$ & 31,2145 & 29,88479 & 200 & calcareus algae & 12 & 3 \\
\hline 157 & plankton1 & Off Dekhela & 31,170397 & 29,743697 & 200 & & 6 & 3 \\
\hline 158 & plankton10 & $\begin{array}{l}\text { Plankton station X, } \\
\text { Alexandria, at st. } \\
26\end{array}$ & 31,386934 & 29,817017 & 200 & & 2 & 2 \\
\hline 159 & plankton12b & $\begin{array}{l}\text { Lake Edku, near } \\
\text { isle Derfil }\end{array}$ & 31,26878 & 30,253991 & 200 & mud & 1 & 1 \\
\hline 160 & plankton14 & $\begin{array}{l}\text { Plankton station } \\
\text { XIV, Alexandria, at } \\
\text { st54 }\end{array}$ & 31,456747 & 29,955787 & 200 & & 2 & 1 \\
\hline
\end{tabular}




\begin{tabular}{|c|c|c|c|c|c|c|c|c|}
\hline 161 & plankton16 & $\begin{array}{l}\text { Plankton XVI, } \\
\text { Alexandria, at st.64 }\end{array}$ & 31,325667 & 29,710205 & 200 & & 2 & 2 \\
\hline 162 & plankton17 & $\begin{array}{l}\text { At st. 117, North of } \\
\text { Abu Talat, Egypt }\end{array}$ & 31,257007 & 29,660777 & 200 & $\begin{array}{l}\text { sandy, muddy, } \\
\text { Caulerpa, Halimeda }\end{array}$ & 2 & 2 \\
\hline 163 & plankton3 & $\begin{array}{l}\text { Off the Eastern } \\
\text { Harbour }\end{array}$ & 31,22687 & 29,892666 & 200 & & 3 & 2 \\
\hline 164 & plankton4a & $\begin{array}{l}\text { North of Abu Qir } \\
\text { Bay }\end{array}$ & 31,363663 & 30,016892 & 200 & stony mud bottom & 2 & 2 \\
\hline 165 & plankton6 & $\begin{array}{l}\text { Lake Mareotis, } \\
\text { near the Mex } \\
\text { Experimental } \\
\text { station }\end{array}$ & 31,143538 & 29,854457 & 1500 & & 1 & 1 \\
\hline 166 & plankton7 & $\begin{array}{l}\text { Lake Mareotis, } \\
\text { eastward, near the } \\
\text { fresh-water fish- } \\
\text { market, at the } \\
\text { coast and further } \\
\text { away from the } \\
\text { coast in the } \\
\text { plankton }\end{array}$ & 31,178765 & 29,91436 & 1500 & & 1 & 1 \\
\hline 167 & Po & $\begin{array}{l}\text { Posidonia bottom, } \\
\text { near the Ras El Tin } \\
\text { Barracks }\end{array}$ & 31,204189 & 29,869984 & 200 & Posidonia & 6 & 3 \\
\hline 168 & POK & $\begin{array}{l}\text { Crags outside the } \\
\text { Barracks of Ras El } \\
\text { Tin, off the } \\
\text { posidonia bottom }\end{array}$ & 31,207036 & 29,86802 & 200 & $\begin{array}{l}\text { crags, Caulerpa, } \\
\text { Halimeda, brown } \\
\text { algae }\end{array}$ & 22 & 4 \\
\hline 169 & SidiBishr & Sidi Bishr & 31,26592 & 29,98614 & 500 & $\begin{array}{l}\text { zone of algae on } \\
\text { rocks }\end{array}$ & 18 & 3 \\
\hline 170 & Silsila & Beach of Silsila & 31,211061 & 29,909446 & 200 & sand & 1 & 1 \\
\hline 171 & $\operatorname{Trawl}(\mathrm{T})$ & $\begin{array}{l}\text { Trawl in Abu Qir } \\
\text { Bay }\end{array}$ & 31,427605 & 30,23248 & 6000 & $\begin{array}{l}\text { muddy ground with } \\
\text { algae }\end{array}$ & 16 & 4 \\
\hline 172 & westernharbour1 & $\begin{array}{l}\text { Western Harbour, } \\
\text { Arsenal Basin, } \\
\text { West Harbour } \\
\text { epifauna }\end{array}$ & 31,194964 & 29,874831 & 800 & & 7 & 3 \\
\hline
\end{tabular}




\section{Difficulties regarding data digitization}

During the digitization process, several issues with the data were encountered. The majority of these problems were similar across datasets. In the following paragraphs, we will highlight the most common ones and explain how they have been dealt with.

1. Data on the same sampling event were scattered and repeated in different publications. This created inconsistencies both across and within publications. Information on stations characteristics and sampling protocol were often repeated with small differences, missings or typographic errors due to different languages in the publications as well as in the preliminary reports. Within each faunistic report, occurrence records were often presented in two different ways, once as a list of species by station and again as a list of stations by species, leading to small differences or typographic errors. For practical reasons, we decided to consider as correct the information on sampling protocol that was obtained from the preliminary reports and the information on species distribution obtained from species list rather than station list. In any case, different information was always kept as a remark in the datasets.

2. The final number of stations recorded was 172: a total of 150 benthic stations reported in the Steuer's preliminary report (1935) enriched with 10 planktonic stations derived from the 12 faunistic reports and with 10 new stations generated during the digitization process. Some stations described only verbally in the faunistic reports were not on a map. For example, some species referred to be collected from the "eastern harbour, on the body of a ship" or "eastern harbour, epifauna" without displaying on the map. In such case, a new station was created (e.g. easterharbour1), in order to include all available information. Other examples were LacMarioutCenter and westernharbour1 stations. In addition, new station IDs had to be created by the data management team because some stations were reported in the historical maps without a station name. For example, a sampling position "near the bath" was mentioned and mapped in many reports without a specific station name. Other stations without a station name were coastAbuQirnearRosetta, LakeEdku_marinebeach, LakeEdkubridge, offSidiBishr and Silsila.

3. Besides general difficulties, described above, some sampling stations needed extra consideration.

- For station D2, the sampling date was not recorded in mollusks report (Steuer 1939b). Nevertheless, this gap was corrected using the 1st of October $1933(1 / 10 / 1933)$ as the sampling date because all the trips were one day trips and in all papers D2 was visited only on that day.

- Another problematic station was station 104. This station was reported in four faunistic reports: two of them without sampling date and the rest with different dates, 1/11/1933 in Sipuncula and 8/11/1933 in Polychaeta. Eventually, the date 8/11/1933 was considered as correct instead of $1 / 11 / 1933$ and used for all the reports. This decision was made, because, as mentioned above, stations were reported in chronological order and 
station 104 was reported under the ones of $7 / 11$ and before the ones of 9/11.

- Some stations were reported with subdivisions. One of them was station 105 that was divided into 105a and 105b in some faunistic reports, but they were not mapped separately. In this case, we used the coordinates of station 105 with higher uncertainty for both subdivisions. In the report on mollusks, and specifically regarding the species Cerithium vulgatum and Tricolia pullus, the stations $105 \mathrm{a}$ and $105 \mathrm{~b}$ were displayed on two maps in opposite locations (Steuer 1939b, figures 5 and 8 in pp.15 and 24).

- Station 13 was also referred as $13 \mathrm{~b}$ in the report on mollusks (Steuer $1939 \mathrm{~b})$ but it was mapped as station 13 and as a result we used the coordinates of station 13.

4. In some planktonic hauls it was difficult to distinguish sampling and station depth. For example, "...over about 15 fathoms 3 vertical hauls out of depths of $20 \mathrm{~m}$ " was the description of station plankton4a in the Sipuncula report (Steuer 1939a). Thus, it was assumed, in accordance with preliminary reports, that station depth was 15 fathoms (about $28 \mathrm{~m}$ ) and sampling depth was 0-20 $\mathrm{m}$.

5. In a few cases, specimens that were reported in the historical publications could not be taxonomically updated due to missing species name authorship or due to ambiguous names. For example, Heliacus moniliferus was recorded in the Mollusca report (Steuer 1939b), but actually this name is used for a fossil, not a living animal as reported in Steuer (1939b).

6. Original sampling date and sampling depth were not recorded in the historical papers concerning Echinodermata and Ascidiacea. During the digitization of all the datasets concerning the Egypt Expedition, it was clear that in most of the cases, sampling dates and sampling depths were the same as in other datasets of Egypt Expedition and as a result date and depth information were taken from other faunistic reports and the preliminary report.

7. Finally, maps were only available as a .pdf in the preliminary report so they were converted to a .jpg or .tiff, in order to proceed to integration in GIS and be georeferenced. Maps recorded the geographical information of the sampling stations. Maps were not ideally suited for georeferencing: they were not accurate, had no scale or map projection system, and the accuracy of the scanner which had created the digitized version was unknown. The original map had to be aligned with their actual geographical location, by linking each point to its equivalent on a modern, accurate digital map (Rumsey and Williams 2002). In the end, Geographic Coordinate System: GCS_WGS_1984 and Datum: D_WGS_1984 was defined as the geographical coordinate system for the maps and the stations were digitized in a new feature class in GIS on a scale of 1:250.000. However it is impossible to perfectly align old maps due to landscape changes over the decades. For example, both the harbor of Alexandria as well as Lake Mariout were significantly extended over the course of the 20th century. A coordinates uncertainty of around 200 meters was estimated by the root mean square (RMS) - this is the georeference error. This value describes how consistent the transformation is between the different control points. In some cases, where the sampling stations were not well defined on the 
maps (lack of station name, landscape changes) new points were placed on the map manually, with higher uncertainty. The planktonic stations were also placed manually on the map.

\section{Geographic coverage}

Description: The Egypt expedition covered, with 162 benthic and 10 planktonic stations, the area along the coasts of Alexandria, the Suez Canal, the Nile river and the lakes Edku and Mariout (Fig. 2).

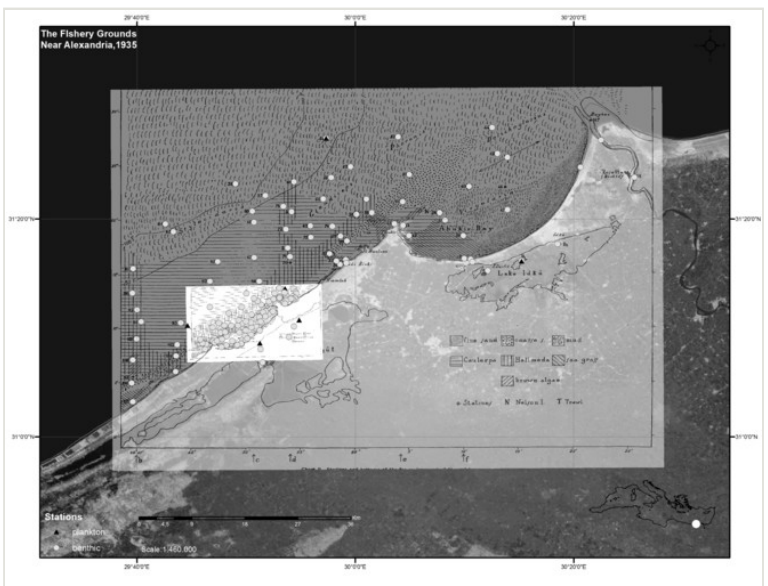

Figure 2.

Georeferenced map of all stations from "The fishery grounds near Alexandria" macrofaunal reports.

Coordinates: 31.054 and 31.473 Latitude; 30.426 and 29.658 Longitude.

\section{Taxonomic coverage}

Description: This set of historical data includes distribution information for 571 marine macrobenthic species belonging to 10 phyla, 21 classes and 257 families (Fig. 3). Malacostraca was the most speciose class with $26 \%$ of total species found, followed by Polychaeta (21\%), Gastropoda (20\%) and Bivalvia (14\%) (Fig. 4). The family with the highest number of species richness was Syllidae (17 species), followed by Trochidae (14 species) and Veneridae (11 species). For the rest of the families, more than half of them (146 of 257) were represented by a single species. 


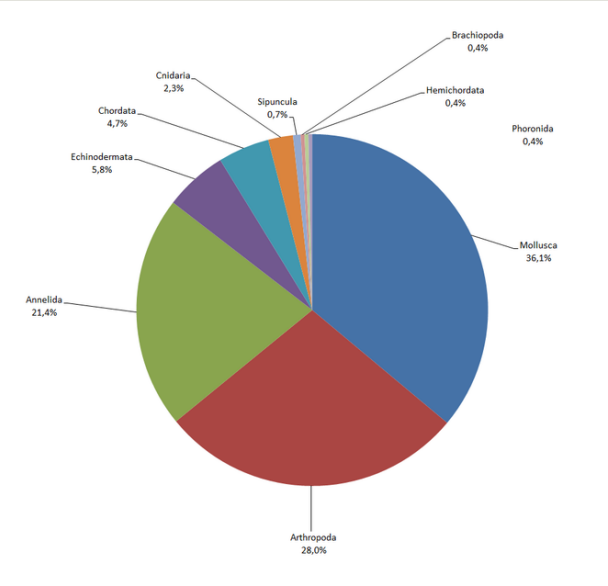

Figure 3.

Percentage of macrofaunal species per phylum.

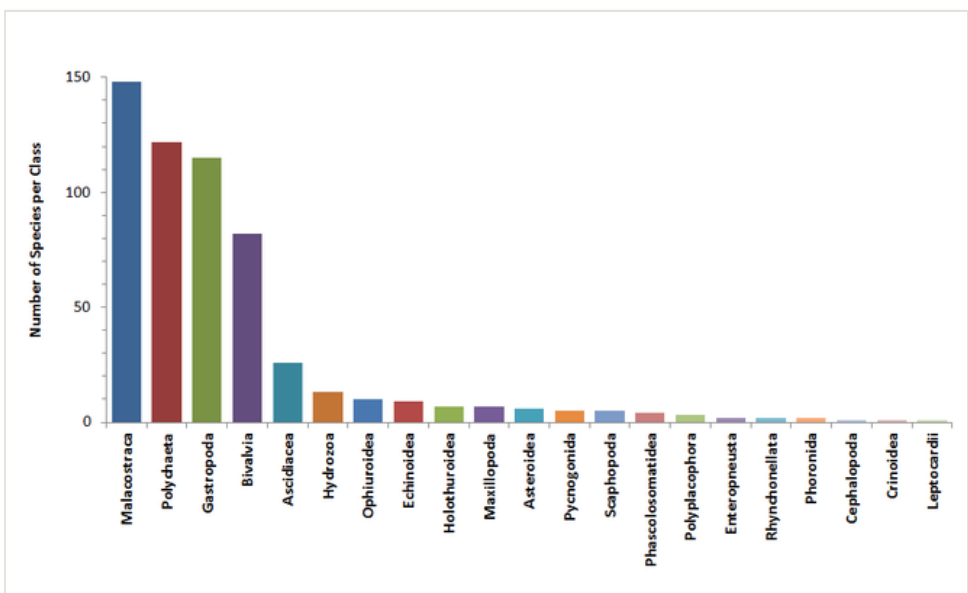

Figure 4.

Number of macrofaunal species per class.

These macrofaunal species were distributed in 172 stations located in the marine area off Alexandria, Egypt (Table 2). Species richness at the different sampling stations was very heterogenous. The most species rich stations were station 61 , located above the isobath of 50 fathoms $(90 \mathrm{~m})$, station 35, off Sidi Bishr and station 7 located close to the Eastern Harbour of Alexandria. The ten most common species in the study area are presented in Table 3. These species were found in more than $10 \%$ of the total number of stations. 
Table 3.

List of the most common species found in more than $10 \%$ of the stations

\begin{tabular}{|c|c|c|c|c|c|c|c|}
\hline Scientific Name & $\begin{array}{l}\text { Scientific } \\
\text { Name } \\
\text { accepted }\end{array}$ & Kingdom & Phylum & Class & Order & Family & $\begin{array}{l}\text { Number } \\
\text { of } \\
\text { stations }\end{array}$ \\
\hline $\begin{array}{l}\text { Dentalium (Antalis) } \\
\text { dentale forma } \\
\text { inaequicostatum }\end{array}$ & $\begin{array}{l}\text { Antalis } \\
\text { inaequicostata }\end{array}$ & Animalia & Mollusca & Scaphopoda & Dentaliida & Dentaliidae & 41 \\
\hline $\begin{array}{l}\text { Branchiostoma } \\
\text { lanceolatum }\end{array}$ & $\begin{array}{l}\text { Branchiostoma } \\
\text { lanceolatum }\end{array}$ & Animalia & Chordata & Leptocardii & & $\begin{array}{l}\text { Branchiosto- } \\
\text { matidae }\end{array}$ & 25 \\
\hline Chlamys glabra & $\begin{array}{l}\text { Flexopecten } \\
\text { glaber }\end{array}$ & Animalia & Mollusca & Bivalvia & Pectinida & Pectinidae & 21 \\
\hline Pilumnus hirtellus & $\begin{array}{l}\text { Pilumnus } \\
\text { hirtellus }\end{array}$ & Animalia & Arthropoda & Malacostraca & Decapoda & Pilumnidae & 21 \\
\hline Columbella rustica & $\begin{array}{l}\text { Columbella } \\
\text { rustica }\end{array}$ & Animalia & Mollusca & Gastropoda & $\begin{array}{l}\text { Neogastro- } \\
\text { poda }\end{array}$ & Columbellidae & 19 \\
\hline Abra ovata & $\begin{array}{l}\text { Abra } \\
\text { segmentum }\end{array}$ & Animalia & Mollusca & Bivalvia & Cardiida & Semelidae & 19 \\
\hline $\begin{array}{l}\text { Glycymeris pilosus } \\
\text { var lineatus }\end{array}$ & $\begin{array}{l}\text { Glycymeris } \\
\text { bimaculata }\end{array}$ & Animalia & Mollusca & Bivalvia & Arcida & Glycymerididae & 19 \\
\hline $\begin{array}{l}\text { Beguina (Glans) } \\
\text { trapezia }\end{array}$ & Glans trapezia & Animalia & Mollusca & Bivalvia & Carditida & Carditidae & 17 \\
\hline $\begin{array}{l}\text { Murex (Truncularia) } \\
\text { trunculus }\end{array}$ & $\begin{array}{l}\text { Hexaplex } \\
\text { trunculus }\end{array}$ & Animalia & Mollusca & Gastropoda & $\begin{array}{l}\text { Neogastro- } \\
\text { poda }\end{array}$ & Muricidae & 17 \\
\hline $\begin{array}{l}\text { Nassarius (Hinia) } \\
\text { incrassatus }\end{array}$ & $\begin{array}{l}\text { Tritia } \\
\text { incrassata }\end{array}$ & Animalia & Mollusca & Gastropoda & $\begin{array}{l}\text { Neogastro- } \\
\text { poda }\end{array}$ & Nassariidae & 17 \\
\hline
\end{tabular}

\section{Taxa included:}

\begin{tabular}{|l|l|}
\hline Rank & Scientific Name \\
\hline phylum & Annelida \\
\hline phylum & Mollusca \\
\hline phylum & Arthropoda \\
\hline class & Hydrozoa \\
\hline phylum & Echinodermata \\
\hline phylum & Sipuncula \\
\hline
\end{tabular}




\begin{tabular}{|l|l|}
\hline class & Ascidiacea \\
\hline phylum & Phoronida \\
\hline phylum & Brachiopoda \\
\hline class & Enteropneusta \\
\hline
\end{tabular}

\section{Temporal coverage}

Data range: $1933401-19331118$.

Notes: Sampling started at April 1st 1933 and ended at November 18th 1933.

\section{Usage rights}

Use license: Creative Commons Public Domain Waiver (CC-Zero)

\section{Data resources}

Data package title: Digitization of historical dataset - Egypt Expedition

\section{Number of data sets: 1}

Data set name: The fishery grounds near Alexandria

Character set: UTF-8

\section{Download URL: http://ipt.medobis.eu/}

\section{Data format: Text}

Description: This publication consists of 12 individual datasets containing different taxonomic groups as published by the original authors between 1935 and 1940 and 2 metadata only (Vatova 1935, Steuer 1935). All datasets are available via the IPT (http:// ipt.medobis.eu/), serving as the Mediterranean node of the Ocean Biogeographic Information System (MedOBIS). Datasets will also be available on OBIS website in winter 2016. Individual download URLs for each dataset are available in Table 1. Table 4, Table 5 and Table 6 describe events, occurrences and measurements or facts, respectively. 
Table 4.

Column description of 'Darwin Core Event' table

\begin{tabular}{|c|c|}
\hline Column label & description \\
\hline id & Same as eventID \\
\hline type & The nature or genre of the resource (dataset etc) \\
\hline eventID & $\begin{array}{l}\text { An identifier for the sampling event (event=sampling that occurs at a place,time, } \\
\text { specific protocol and depth) }\end{array}$ \\
\hline parentEventID & $\begin{array}{l}\text { An identifier for the broader Event at a specific station, that groups this and } \\
\text { potentially other Events }\end{array}$ \\
\hline samplingProtocol & $\begin{array}{l}\text { The name of, reference to, or description of the method or protocol used during an } \\
\text { Event }\end{array}$ \\
\hline eventDate & The date-time or interval during which an Event occurred \\
\hline year & $\begin{array}{l}\text { The four-digit year in which the Event occurred, according to the Common Era } \\
\text { Calendar }\end{array}$ \\
\hline month & The original month in which the Event occurred \\
\hline day & The original day in which the Event occurred \\
\hline habitat & A category or description of the habitat in which the Event occurred \\
\hline fieldNumber & The sample name \\
\hline eventRemarks & Comments or notes about the Event \\
\hline locationID & Station name \\
\hline locality & The name or description of the place \\
\hline minimumDepthInMeters & The lesser sampling depth of a range of depth below the local surface, in meters \\
\hline maximumDepthInMeters & The greater sampling depth of a range of depth below the local surface, in meters \\
\hline locationRemarks & Comments or notes about the station where the sample occurred \\
\hline decimalLatitude & The geographic latitude (in decimal degrees) of the station \\
\hline decimalLongitude & The geographic longitude (in decimal degrees) of the station \\
\hline coordinateUncertaintylnMeters & $\begin{array}{l}\text { The horizontal distance (in meters) from the given decimalLatitude and } \\
\text { decimalLongitude describing the smallest circle containing the whole of the } \\
\text { Location }\end{array}$ \\
\hline
\end{tabular}


Table 5.

Column description of 'Darwin Core Occurrence' table

\begin{tabular}{|c|c|}
\hline Column label & description \\
\hline id & Same as occurrencelD \\
\hline type & The nature of the resource (e.g. dataset) \\
\hline institutionCode & $\begin{array}{l}\text { The name in use by the institution having custody of the object(s) or information referred } \\
\text { to in the record }\end{array}$ \\
\hline collectionCode & $\begin{array}{l}\text { The name, coden, or initialism identifying the collection or data set from which the record } \\
\text { was derived }\end{array}$ \\
\hline basisOfRecord & The specific nature of the data record \\
\hline occurrencelD & A unique identifier for the Occurrence \\
\hline catalogNumber & An identifier for the record within the data set or collection \\
\hline occurrenceRemarks & Comments or notes about the Occurrence \\
\hline individualCount & The number of individuals represented present at the time of the Occurrence \\
\hline $\operatorname{sex}$ & The sex of the biological individual(s) represented in the Occurrence \\
\hline lifeStage & $\begin{array}{l}\text { The age class or life stage of the biological individual(s) at the time the Occurrence was } \\
\text { recorded }\end{array}$ \\
\hline eventID & $\begin{array}{l}\text { An identifier for the sampling event (event=sampling that occurs at a place,time, specific } \\
\text { protocol and depth) }\end{array}$ \\
\hline identifiedBy & $\begin{array}{l}\text { A list of names of people, groups, or organizations who assigned the Taxon to the } \\
\text { subject }\end{array}$ \\
\hline identificationReferences & A list of references (publication, global unique identifier, URI) used in the Identification \\
\hline scientificNameID & The Isid from WORMS \\
\hline scientificName & $\begin{array}{l}\text { The full scientific name. When forming part of an Identification, this should be the name } \\
\text { in lowest level taxonomic rank that can be determined }\end{array}$ \\
\hline kingdom & The full scientific name of the kingdom in which the taxon is classified \\
\hline phylum & The full scientific name of the phylum or division in which the taxon is classified \\
\hline class & The full scientific name of the class in which the taxon is classified \\
\hline order & The full scientific name of the order in which the taxon is classified \\
\hline family & The full scientific name of the family in which the taxon is classified \\
\hline genus & The full scientific name of the genus in which the taxon is classified \\
\hline subgenus & $\begin{array}{l}\text { The full scientific name of the subgenus in which the taxon is classified. Values should } \\
\text { include the genus to avoid homonym confusion }\end{array}$ \\
\hline
\end{tabular}




\begin{tabular}{|l|l|}
\hline specificEpithet & The name of the first or species epithet of the scientificName \\
\hline scientificNameAuthorship & $\begin{array}{l}\text { The authorship information for the scientificName formatted according to the } \\
\text { conventions of the applicable nomenclaturalCode }\end{array}$ \\
\hline nomenclaturalCode & The nomenclatural code under which the scientificName is constructed \\
\hline taxonRemarks & Comments or notes about the taxon or name \\
\hline
\end{tabular}

Table 6.

Column description of 'Darwin Core Extended Measurement or Facts' table

\begin{tabular}{|c|c|}
\hline Column label & description \\
\hline eventID & $\begin{array}{l}\text { An identifier for the sampling event (event=sampling that occurs at a place, time, } \\
\text { specific protocol and depth) }\end{array}$ \\
\hline measurementID & An identifier for the MeasurementOrFact \\
\hline measurementType & The nature of the measurement, fact, characteristic, or assertion \\
\hline measurementValue & The value of the measurement, fact, characteristic, or assertion \\
\hline measurementUnit & The units associated with the measurementValue \\
\hline occurrencelD & A unique identifier for the Occurrence \\
\hline measurementMethod & $\begin{array}{l}\text { A description of or reference to (publication, URI) the method or protocol used to } \\
\text { determine the measurement, fact, characteristic, or assertion }\end{array}$ \\
\hline measurementDeterminedBy & $\begin{array}{l}\text { A list of names of people, groups, or organizations who determined the value of the } \\
\text { MeasurementOrFact }\end{array}$ \\
\hline measurementTypeID & An identifier for the measurementType (global unique identifier, URI) \\
\hline measurementAccuracy & The description of the potential error associated with the measurementValue \\
\hline measurementRemarks & Comments or notes accompanying the MeasurementOrFact \\
\hline
\end{tabular}

\section{Additional information}

\section{Conclusions}

Data rescue is an increasing need with expected effects on the scientific and societal perception of biodiversity. Despite the many challenges encountered during the digitization process of historical datasets (e.g. taxonomic updates, georeferencing, misspellings of taxa and places, compiling overlapping information from different publications), the outcome clearly shows that such initiatives are invaluable in making accessible previously unavailable biodiversity data. 
Concerning the Egypt expedition, this paper is the first step for the digitization of the whole set of publications from "The fishery grounds near Alexandria". In Eastern Mediterranean, these data could be used to set the reference conditions for checking the invasion of alien species through the Suez Canal or to compare past species occurrences with current ones. In addition, the availability of these historical data through public databases (such as LifewatchGreece Research Infrastructure and MedOBIS) provides useful tools for present observations or monitoring potential change in benthic communities. Through virtual labs, scientists or other users could search, visualize on a map, combine and download species occurrences from all over the Mediterranean in several different formats.

Digitizing historical datasets offers also valuable information on functional species traits, as they usually contain individual characteristics, such as maturity and body length, and habitat characteristics, such as sediment type and vegetation. Information on functional species traits is required in describing species patterns and assessing future evolution of benthic communities.

\section{Acknowledgements}

This work was supported by the LifeWatchGreece infrastructure, funded by the Greek Government under the General Secretariat of Research and Technology (GSRT), ESFRI Projects, Structural Funds, OPCE II. The authors would like to thank Sarah Faulwetter for her invaluable assistance, training throughout the digitization process and manuscript revision and Christos Arvanitidis for comments on the manuscript. Thanks are also due to 2 reviewers hor helpful comments and critisism on the manuscript. Finally, we thank the European Marine Observation and Data Network - Biology (EMODnet Biology) WP4 partners for inspiration regarding data rescue.

\section{Author contributions}

I. Tsikopoulou and S. Nikolopoulou contributed equally to this work. I. Tsikopoulou, S. Nikolopoulou and A. Legaki were resource creators and content providers. P.D. Dimitriou and E. Avramidou were content providers. I. Tsikopoulou and S. Nikolopoulou wrote the first version of the manuscript. Nicolas Bailly has checked difficult taxonomic cases. All authors contributed to revisions.

\section{References}

- Borja Á, Dauer D, Grémare A (2012) The importance of setting targets and reference conditions in assessing marine ecosystem quality. Ecological Indicators 12 (1): 1-7. http s://doi.org/10.1016/j.ecolind.2011.06.018 
- $\quad$ Costello M, Michener W, Gahegan M, Zhang Z, Bourne P (2013a) Biodiversity data should be published, cited, and peer reviewed. Trends in Ecology \& Evolution 28 (8): 454-461. https://doi.org/10.1016/..tree.2013.05.002

- Costello M, Bouchet P, Boxshall G, Fauchald K, Gordon D, Hoeksema B, Poore GB, RMvS, Stöhr S, Walter TC, Vanhoorne B, Decock W, Appeltans W (2013b) Global Coordination and Standardisation in Marine Biodiversity through the World Register of Marine Species (WoRMS) and Related Databases. PLoS ONE 8 (1): e51629. https:// doi.org/10.1371/journal.pone.0051629

- $\quad$ Fauvel P (1937) Les fonds de peche pres d'Alexandrie. XI Annelides Polychetes. Notes et Memoires No 19. Direction des Recherches des Pecheries, Egypt, 62 pp.

- Fox M (1926) General part. Zoological results of the Cambridge Expedition to the Suez Canal, 1924. Transact. Zool. Soc., London, 22 pp.

- Rumsey D, Williams M (2002) Historical Maps in GIS. In: Knowles AK (Ed.) Past Time, Past Place: GIS for History.

- Schmidt J (1912) Report on the Danish Oceanographical Expeditions 1908-1910 on the Mediterranean and Adjacent Seas. Andr. Fred. Host \& Son, Copenhagen, 51 pp.

- $\quad$ Steuer A (1935) The fishery grounds near Alexandria. I. Preliminary report. Notes and Memoirs No 8. Fisheries Research Directorate, Egypt, 24 pp.

- Steuer A (1939a) The fishery grounds near Alexandria. XVIII Sipunculoidea, Phoronidea, Brachiopoda, Enteropneusta and Acrania. Notes and Memoirs No 33. Hydrobiology and Fisheries Directorate, Egypt, $15 \mathrm{pp}$.

- $\quad$ Steuer A (1939b) The fishery grounds near Alexandria. XIX Mollusca. Notes and Memoirs No 38. Hydrobiology and Fisheries Directorate, Egypt, 152 pp.

- Vatova A (1935) The fishery grounds near Alexandria. II. A bottom sample taken at Alexandria. Notes and Memoirs No. 9. Fisheries Research Directorate, Egypt, 4 pp.

- Wieczorek J, Braak K (2015) The GBIF Integrated Publishing Toolkit User Manual, version 2.3. Copenhagen: Global Biodiversity Information Facility, 57 pp.

- WoRMS Editorial Board (2016) World Register of Marine Species. http:// www.marinespecies.org/aphia.php?p=match. Accession date: 2016719.

\section{Supplementary material}

\section{Suppl. material 1: Extended Measurement or Facts}

Authors: Irini Tsikopoulou, Stamatina Nikolopoulou, Aglaia Legaki

Data type: Measurements

Brief description: Body length measurements are available for the following datasets:

1. EgyptExpeditionCumaceaStomatopodaLeptostacaea

2. EgyptExpeditionPolychaeta

3. EgyptExpeditionAmphipoda

4. EgyptExpeditionTanaidacealsopoda

5. EgyptExpeditionMollusca

Carapace length measurements are available for the dataset EgyptExpeditionDecapoda

Filename: measurements_Egypt_IPT.csv - Download file $(24.62 \mathrm{~kb})$ 